\title{
A Case of Nutritional Secondary Hyperparathyroidism in a Siberian Tiger Cub
}

\author{
Dong-Sun $\mathrm{WON}^{1)}$, Chul PARK ${ }^{1)}$, Young-Joo $\mathrm{IN}^{1)}$ and Hee-Myung PARK ${ }^{1) *}$ \\ ${ }^{1)}$ Department of Veterinary Internal Medicine, College of Veterinary Medicine, Konkuk University, \#1 Hwayang-dong Kwang-gin-gu, \\ Seoul, South Korea 143-701
}

(Received 14 August 2003/Accepted 12 December 2003)

ABSTRACT. A three-month-old female Siberian tiger cub with hindlimb ataxia was referred to the veterinary teaching hospital of Konkuk University. The patient was fed only beef without supplementation of calcium and vitamins after weaning. The tiger was presented with ataxia and back pain on digital palpation. In addition, abnormal gait, reluctance to move, and depressed withdrawal reflex were noted at the neurological examination. The overall osteodystrophic change of the lumbosacral vertebrae was observed on the lateral and ventrodorsal view of radiographic examination. And also PTH level was increased in hormonal assay when compared to that of cat reference range. Based on the results of examinations, nutritional secondary hyperparathyroidism was diagnosed. Clinical signs of this patient were improved after administration of vitamin $\mathrm{D}$ and calcium. This case demonstrates that nutritional hyperparathyroidis $\mathrm{m}$ could be occurred in wild animals raised on a meat diet containing imbalanced calcium and phosphate.

KEY WORDS: calcium, hyperparathyroidism, Siberian tiger.

J. Vet. Med. Sci. 66(5): 551-553, 2004

Nutritional secondary hyperparathyroidsim is caused by diets low in calcium and high in phosphorous, while a deficiency of vitamin D may also be incriminated [5]. Control of calcium metabolism and in particular the extracellular level of ionized calcium is mediated through the action of PTH, calcitonin, and vitamin D. Dietary calcium deficiency, such as beef or liver, results in transient decreased serum calcium concentration, and inducing increased PTH secretion [6]. As a result, the calcium absorption from gastrointestinal (GI) tract and bone is increased, enhancing phosphorus excretion by kidney tubules. This can result in severe skeletal disturbance.

In the dog, the most commonly encountered nutritional bone disease is nutritional secondary hyperparathyroidism [1]. This endocrinopathy is primarily of importance in the dog but is occasionally seen in kittens and other species [8, 9]. In carnivores it is seen in young rapidly growing individuals where it is also referred to as juvenile osteoporosis, while in equines it occurs in adults where it is also referred to as bighead, bran disease or miller's disease.

This is presented to show that nutritional secondary hyperparathyroidism in the Siberian tiger cub raised on a diet with calcium and phosphorous imbalance could be occurred.

A three-month-old female Siberian tiger cub with acute bilateral hindlimb paresis was referred to the veterinary teaching hospital of Konkuk University (Fig. 1). On history taking, the tiger was fed only beef amounts of 6 to $12 \%$ of body weight after weaning. She showed acute lameness after walking up the hill. Physical examination revealed mild muscular shivering and back pain, in particular around lumbosacral segment. In addition, the tiger showed abnormal gait and reluctance to move. The patient showed

\footnotetext{
* Correspondence to: Park, H.-M., Department of Veterinary Internal Medicine, College of Veterinary Medicine, Konkuk University, \#1 Hwayang-dong Kwangjin-gu Seoul, Korea 143-701.
}

depressed $(+1$ : Grades are assigned as follows: $0=$ no response $;+1=$ a decreased response,$+2=$ a normal response; $+3=$ an exaggerated response; and $+4=$ a myotatic reflex with clonus) withdrawal reflex and quadriceps reflex in the neurological examination.

Radiographically, the radiodensity of the lumbar vertebra was decreased at the lateral and ventrodorsal view, the affected bone opacity was similar to that of soft tissue (Fig. 2 ). Based on history taking and radiographic findings, acute hindlimb paresis may be induced by severe movement (eg, walking up the hills). However, complete blood count (CBC) profiles exhibited no remarkable findings. Serum chemistry profiles revealed increased alkaline phosphatase (ALP) (113; normal range 8-55 u/L), creatinine phosphokinase (CK) (381; normal range 32-311 u/L) and inorganic phosphorus level (7.9; normal range $2.5-7.5 \mathrm{mg} / \mathrm{d} l$ ). However, this finding was not typical in nutritional secondary hyperparathyrodism because results of this serum chemistry in rapidly growing animals are usually considered to be normal.

Results of hormonal assay showed elevated PTH (Active Intact PTH, DSL-800, USA) level (41.8; reference range 3$25 \mathrm{pg} / \mathrm{ml}$ ) when compared to that of cat reference range [2].

Treatment consisted of cage confinement and supplementation with vitamin $\mathrm{D}$ and calcium in diets.

Control of calcium metabolism is mediated through the action of PTH, calcitonin, and vitamin D. In addition, corticosteroids, glucagon, thyroxine, estrogens and somatotropin may influence calcium homeostasis [5]. Of all, the main function of PTH is maintenance of normal serum calcium concentration by action on bone, kidney, and intestinal mucosa. And it also enhances phosphorus excretion by kidney tubules, which assist in maintaining the serum calcium to phosphorus ratio. Chronic hypocalcemia due to dietary calcium deficiency leads to skeletal demineralization or excess bone resorption [6]. Thus, nutritional secondary 


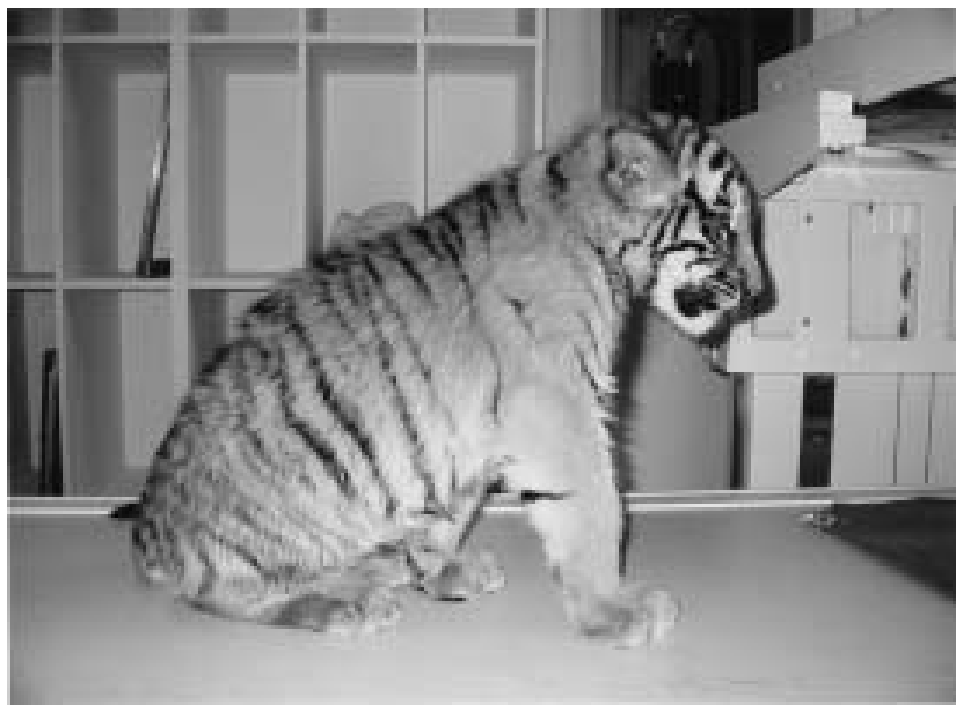

Fig. 1. A three-month-old female Siberian tiger cub shows ataxia and acute bilateral hindlimb paresis.

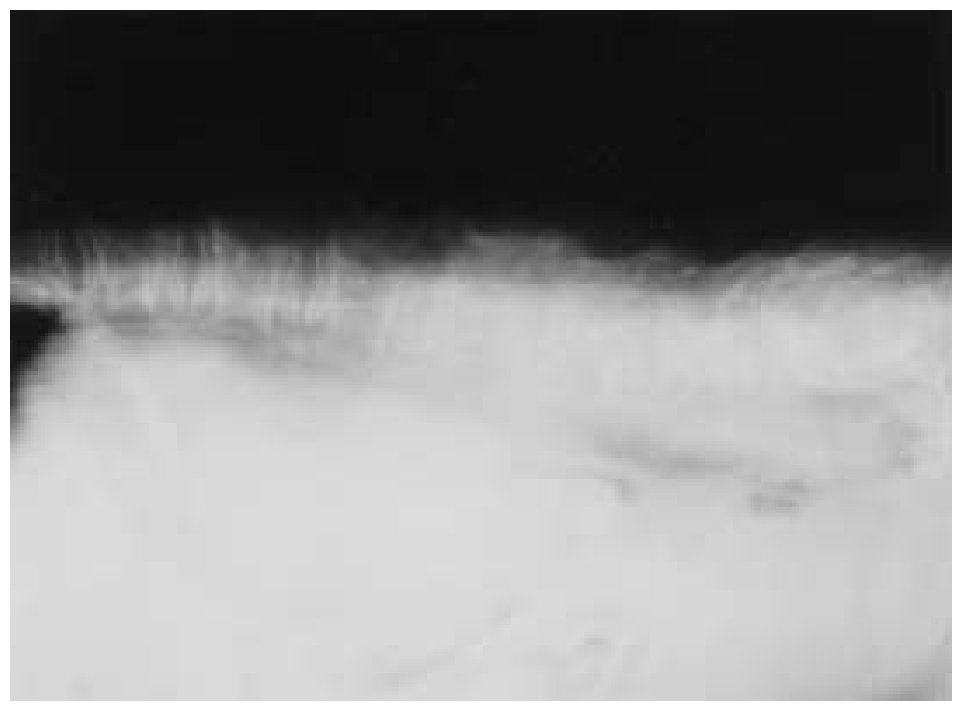

Fig. 2. Lateral view of the lumbar vertebrae of a Siberian tiger cub with nutritional hyperparathyroidism. The overall opacity of the skeleton is decreased, and vertebrae are misshapen.

hyperparathyroidism, a metabolic disorder, could be induced, resulting in osteopenia from excessive bone resorption. This is the most commonly encountered nutritional bone disease and is primarily of importance in dogs and cats but is occasionally reported in the wild animals, especially in carnivores with all-meat diet $[8,9]$.

Based on the history taking, tiger cub in this case has been fed only beef without supplementation of calcium and vitamin $\mathrm{D}$ in diet. It may be caused by diets providing excess phosphate with or without low calcium and this imbalance induces hypocalcemia, which increases releasing of PTH. Thus this dietary management in wild animals may lead to nutritional secondary hyperparathyroidism and enhance calcium resorption from bone, which results in osteodystrophic change in lumbosacral vertebrae. This finding is consistent with the present case. Thus, unless sufficient diet is provided continuously, it causes progressive skeletal demineralization and bone disorders.

According to the previous reports [4], clinical signs in affected young animals or adults are lameness, reluctance to stand or walk, poor growth, posterior paresis and skeletal pain. Costochondral junctions and metaphysis appear swollen. Hematological examination is less confined value in confirming nutritional hyperparathyroidism. The serum cal- 
cium concentration is often revealed normal because of compensatory changes. Serum inorganic phosphorus concentration and alkaline phosphatase may appear high but should be interpreted carefully because of high value in growing animals. As one report [7], cats that diagnosed as nutritional secondary hyperparathyroidism showed markedly increased serum parathormone levels and vitamin $\mathrm{D}(1,25(\mathrm{OH}) 2$-vitamin $\mathrm{D} 3 ; 25(\mathrm{OH})$-vitamin $\mathrm{D} 3)$ in the laboratory findings. Results of hematological and serum chemistry profiles are consistent with those described above in this case.

Radiographically, osteodystrophic change in this case was observed without fracturing. In addition, the bone opacity was similar to that of soft tissue. However, spinal deformity and pathologic folding fractures of appendicular and the axial skeleton were not noted [9].

Affected tiger cub was confined to reduce the risk of fractures and deformity. And balanced nutritional diets were fed with supplementation of calcium and vitamin D in diet. A balanced diet and protective cage rest resulted in quick clinical recovery in this case.

In conclusion, this case demonstrates that nutritional secondary hyperparathyroidism can be occurred in rapidly growing wild animals that are fed all-meat diets (low cal- cium and high phosphorus) after weaning. However, if appropriate management is intervened, clinical signs recovered quickly and nutritional secondary hyperparathyroidism can be manageable.

ACKNOWLEDGEMENT. This work was supported by a grant from Konkuk University.

\section{REFERENCES}

1. Bennett D. 1976. Vet. Rec. 98: 313-321.

2. Carmel, T. M. and Andrew, G. T. 1998. p. 243. Manual of Small Animal Endocrinology, 2nd ed., BSAVA.

3. Kealy, R. D., Lawler, D. F. and Monti, K. L. 1991. J. Nutr. 121: 66-69.

4. Lourens, D. C. 1980. J. S. Afr. Vet. Assoc. 51: 121-123.

5. Reiland, S. 1978. Acta Radiol. (Suppl.) 358: 91-105.

6. Schoenmakers, I., Hazewinkel, H. A., Voorhout, G., Carlson, C.S. and Richardson, D. 2000. Vet. Rec. 147: 652-660.

7. Tomsa, K., Glaus, T., Hauser, B., Fluckiger, M., Arnold, P., Wess, G. and Reusch, C. 1999. Small Anim. Pract. 40: 533539.

8. Van Pelt, R. W. and Caley, M. T. 1974. J. Wild Dis. 10: $47-$ 52.

9. van Rensburg, I. B. and Lowry, M. H. 1988. J. S. Afr. Vet. Assoc. 59: 83-86. 